\title{
Novel Arterial Pathology in Mice and Humans Hemizygous for Elastin
}

\author{
Dean Y. Li, ${ }^{*}$ Gilles Faury, $\|$ Douglas G. Taylor, ${ }^{\prime}$ Elaine C. Davis, ${ }^{\star \star}$ Walter A. Boyle, ${ }^{\natural}$ Robert P. Mecham, $\|$ Peter Stenzel, ${ }^{\ddagger}$ \\ Beth Boak, ${ }^{\S}$ and Mark T. Keating ${ }^{\ddagger \S}$ \\ * Cardiology Division and Program in Human Molecular Biology and Genetics, University of Utah Health Sciences Center, ${ }^{\ddagger}$ Department \\ of Human Genetics, Eccles Institute of Human Genetics, ${ }^{\S}$ Howard Hughes Medical Institute, Salt Lake City, Utah 84112-5330;

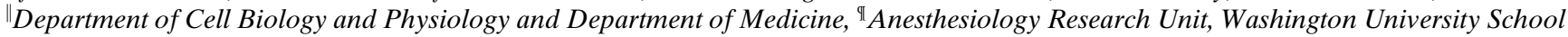 \\ of Medicine, St. Louis, Missouri 63110; **Department of Cell Biology and Neuroscience, University of Texas Southwestern Medical \\ Center, Dallas, Texas 75235-9039; and ${ }^{\ddagger}$ Department of Pathology, Oregon Health Sciences University, Portland, Oregon 97201
}

\begin{abstract}
Obstructive vascular disease is an important health problem in the industrialized world. Through a series of molecular genetic studies, we demonstrated that loss-of-function mutations in one elastin allele cause an inherited obstructive arterial disease, supravalvular aortic stenosis (SVAS). To define the mechanism of elastin's effect, we generated mice hemizygous for the elastin gene $(E L N+/-)$. Although $E L N$ mRNA and protein were reduced by $50 \%$ in $E L N+I-$ mice, arterial compliance at physiologic pressures was nearly normal. This discrepancy was explained by a paradoxical increase of $35 \%$ in the number of elastic lamellae and smooth muscle in ELN $+I-$ arteries. Examination of humans with $E L N$ hemizygosity revealed a 2.5 -fold increase in elastic lamellae and smooth muscle. Thus, ELN hemizygosity in mice and humans induces a compensatory increase in the number of rings of elastic lamellae and smooth muscle during arterial development. Humans are exquisitely sensitive to reduced $E L N$ expression, developing profound arterial thickening and markedly increased risk of obstructive vascular disease. (J. Clin. Invest. 1998. 102:1783-1787.) Key words: elastin - cardiovascular models - mice, transgenic • vascular disease $\cdot$ compliance
\end{abstract}

\section{Introduction}

Obstructive vascular disease is the predominant cause of mortality and morbidity in developed nations (1). Extensive research has implicated key pathogenic roles for lipids and growth factors, but it is likely that other factors participate in the pathogenesis of these disorders. In a series of molecular genetic experiments, we demonstrated that a human obstructive vascular disorder, supravalvular aortic stenosis (SVAS), is associated with hemizygosity of the elastin gene (ELN) (2$6)$. Mutations in genes encoding other structural proteins, in-

Address correspondence to Dean Li, Program in Human Molecular Genetics and Biology, University of Utah, 15N 2030E Suite 2100, Salt Lake City, UT 84112-5330. Phone: 801-581-8909; FAX: 801-585-7423; E-mail: dean@howard.genetics.utah.edu

Received for publication 6 July 1998 and accepted in revised form 16 September 1998.

1. Abbreviations used in this paper: ELN, elastin gene; SVAS, supravalvular aortic stenosis.

J. Clin. Invest.

(C) The American Society for Clinical Investigation, Inc. 0021-9738/98/11/1783/05 \$2.00

Volume 102, Number 10, November 1998, 1783-1787

http://www.jci.org cluding collagen and fibrillin, have also been associated with vascular disease, but these genetic abnormalities cause dissection and degeneration, not obstruction $(7,8)$. The pathogenic mechanisms underlying ELN mutations are not understood.

Elastin is the dominant arterial extracellular matrix protein, comprising $50 \%$ of the dry weight of the aorta (9). Encoded by a single gene on human chromosome 7q11.23, elastin expression is largely confined to the third trimester of fetal development and early postnatal years. Elastin is synthesized by smooth muscle, secreted as a soluble monomer, tropoelastin, and organized into insoluble polymers that form concentric rings of elastic lamellae around the arterial lumen. Each elastic lamella alternates with a ring of smooth muscle, forming a lamellar unit. Elastic lamella provides the resilience that arteries need to absorb hemodynamic stress of cardiac systole and to release this energy in the form of sustained blood pressure during diastole. The number of lamellar units is thought to be species specific, fixed, and genetically predetermined $(10,11)$.

Previously, human pathologic studies of SVAS described medial necrosis, fibrosis, and disorganization, a common endstage pathology of many vascular diseases $(12,13)$. Identifying the mechanism of disease through these studies was hampered by the inability to separate cause and effect. To define the pathogenic mechanism underlying SVAS, we characterized mice hemizygous for ELN. This work demonstrates that the number of lamellar units is neither fixed nor species specific, but is modulated by the level of ELN expression during development. We show that humans are extremely sensitive to reduced $E L N$ expression, developing profound thickening of the arterial wall.

\section{Methods}

Northern analysis. Poly (A) ${ }^{+}$RNA was extracted from the visceral organs of the thorax from mice at birth using a Micro-Fast Tract Kit (Invitrogen, Carlsbad, CA). RNA was electrophoresed on a 1.0\% denaturing agarose gel, transferred to Hybond filter (Amersham, Arlington Heights, IL), and hybridized with a ${ }^{32} \mathrm{P}$-labeled $0.85-\mathrm{kb}$ fragment of mouse $E L N$ cDNA. Filters were rehybridized with a $1.5-\mathrm{kb}$ fragment of human cardiac actin cDNA. Intensity of bands was measured by PhosphorImager analysis. ELN expression in $E L N+/+$ and $E L N+/-$ mice was standardized to cardiac actin expression and compared.

Electron microscopy. Ascending thoracic aortae were dissected from mouse pups at birth after cardiac perfusion with $3 \%$ glutaraldehyde. Aortic segments were sequentially stained with osmium tetroxide, tannic acid, and uranyl acetate, then dehydrated and embedded in Epon. Thin sections (60-nm) were counterstained with uranyl acetate and lead citrate and examined on a Jeol 1200 electron microscope (14).

Histological examination. Mice were fixed overnight in either $4 \%$ paraformaldehyde or methyl Carnoys at $4^{\circ} \mathrm{C}$ and embedded in paraffin. Sections were stained with hematoxylin and eosin and Hart stain for elastin. Individuals blinded to the genotype counted lamellar units 


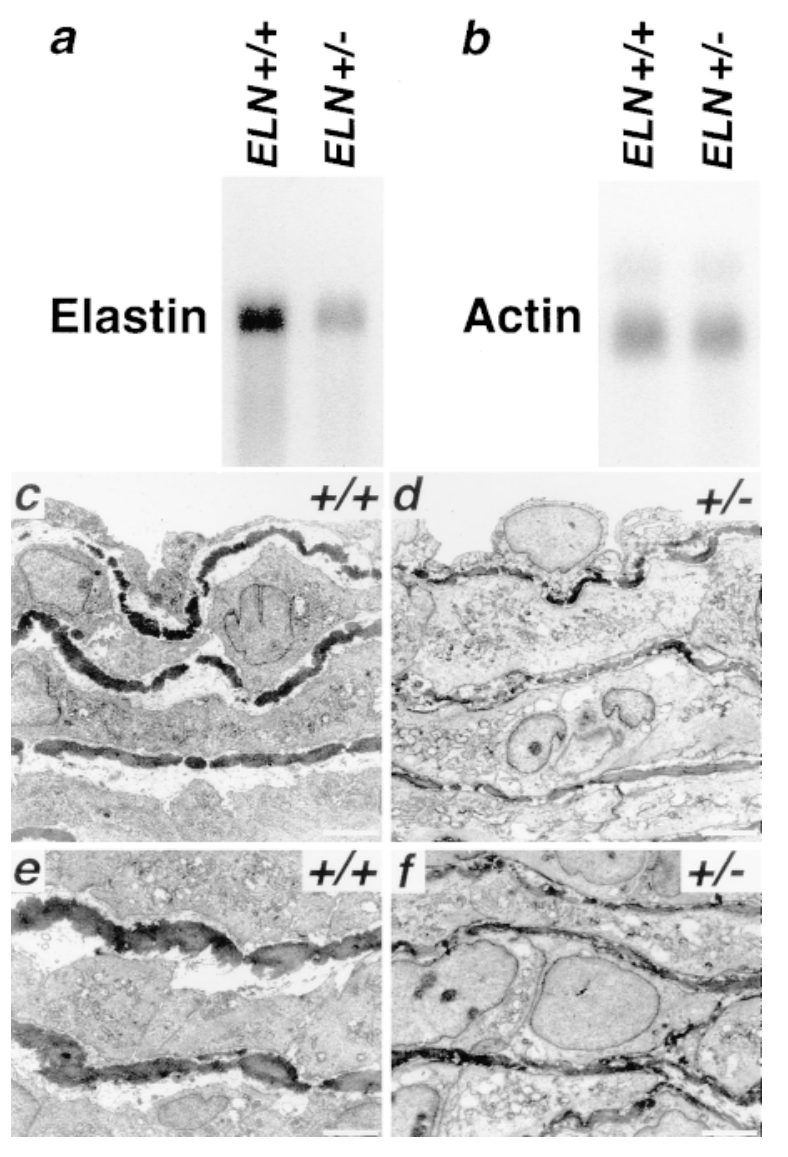

Figure 1. $(a$ and $b)$ Northern analysis of mRNA from $E L N+/+$ and $E L N+/-$ mice at birth for $E L N(a)$ and cardiac actin $(b)$ expression. There is a $47 \%$ decrease in ELN mRNA in $E L N+/-$ mice by PhosphorImager analysis. $(c-f)$ Electron micrographs of ascending aortic cross-sections from $E L N+/+(c$ and $e)$ and $E L N+/-(d$ and $f)$ mice at birth. Subendothelial ( $c$ and $d$ ) and medial sections ( $e$ and $f$ ) for both genotypes are compared. In $E L N+/+$ aortae, circumferentially oriented smooth muscle cells are interposed between well developed elastic lamellae. Note that elastic lamellae are abnormal and $\sim 50 \%$ thinner in $E L N+/-$ aortae. Bar, $3.0 \mu \mathrm{m}$.

on two separate occasions. The statistical significance was calculated by comparison of the means using $t$ test analysis.

Vascular extensibility. Arteries studied were cannulated and mounted on the pressure myograph (15). The vessel was transilluminated under an inverted microscope connected to a CCD camera, allowing the continuous recording of the outer diameter of the vessel. In the ascending aorta, intravascular pressure was increased from 75 to $175 \mathrm{mmHg}$ by steps of $25 \mathrm{mmHg}(0-50 \mathrm{mmHg}$ by steps of 10 $\mathrm{mmHg}$ for the left pulmonary artery), and the arterial diameter was recorded. Extensibility was calculated using the following formula with $125 \mathrm{mmHg}$ as an example: extensibility $=[($ diameter at 150 $\mathrm{mmHg}$ - diameter at $100 \mathrm{mmHg}) /($ diameter at $100 \mathrm{mmHg})] \times 100$ (16). Diameter statistical analysis was assessed by a four-way ANOVA followed by least significance difference test for postANOVA paired comparisons. Extensibility statistical analysis was assessed by the nonparametric Mann-Whitney $U$ test. $P$ values below 0.05 were considered statistically significant.

\section{Results}

To define the role of $E L N$ hemizygosity in arterial disease, we generated mice hemizygous $(E L N+/-)$ for an $E L N$ null mu-

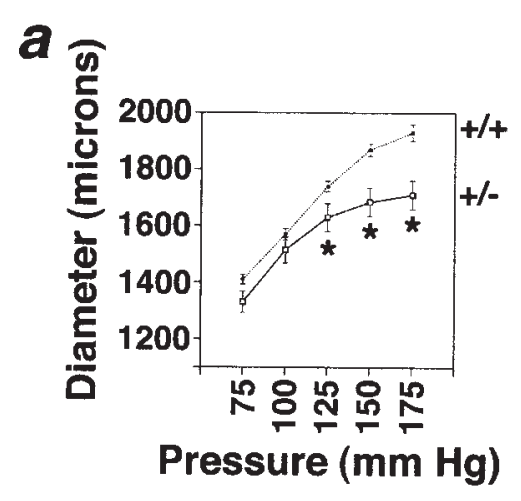

Figure 2. Extensibility of $E L N+/+$ and $E L N$ $+/-$ aortae. (a) Pressure-diameter curve. At pressures $100 \mathrm{mmHg}$ and below, $E L N+1+$ aortae (filled circles) have diameters similar to $E L N+/-$ aortae (open squares). At pressures above 100 $\mathrm{mmHg}$, the diameters of $E L N+/$ - aortae are significantly less than $E L N+/+$ aortae. (b) Extensibility at varying pressures. The extensibilities of $E L N+/+$ (black bars) and ELN

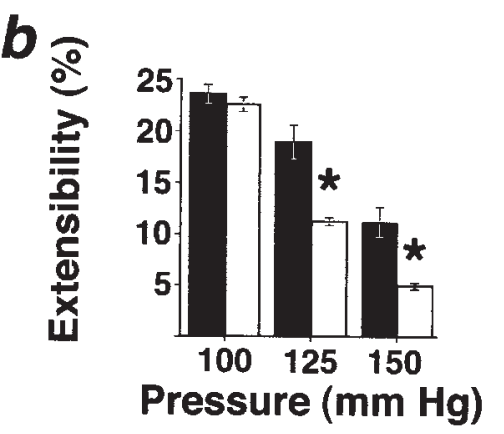

$+/-$ aortae (white bars) are similar at physiologic pressure (100 $\mathrm{mmHg}$ ). However, at elevated pressures (125 and $150 \mathrm{mmHg}$ ) extensibility of $E L N+/-$ aortae is significantly decreased compared with controls. *Statistically significant differences, $P<0.05$.

tation using homologous recombination in embryonic stem cells (17). Three independent recombinant cell lines containing a $4.0-\mathrm{kb}$ deletion of the promoter and exon 1 of $E L N$ were used to generate chimeras. Chimeras were mated to generate $E L N+/-$ mice. $E L N+/-$ mice were identical to $E L N+/+$ mice in gross appearance, behavior, and life expectancy. Northern analysis of $E L N+/-$ mice revealed a $47 \%$ decrease in $E L N$ mRNA when compared with $E L N+/+$ mice at birth (Fig. 1, $a$ and $b$ ). To determine if the structure of elastic lamellae in $E L N+/-$ mice was affected by reduced $E L N$ expression, we examined aortic cross-sections by electron microscopy. Elastic lamellae in $E L N+/-$ aortae (Fig. 1, $d$ and $f$ ) were $\sim 50 \%$ thinner than those in $E L N+/+$ aortae (Fig. 1, $c$ and $e$ ). These data indicate that elastic lamellae in $E L N+/-$ mice are structurally abnormal.

To determine the physiologic consequences of structural changes observed in $E L N+/-$ mice, we measured aortic diameter and extensibility at varying intraluminal pressures. Despite differences in ELN mRNA expression and protein deposition, at a physiologic pressure of $100 \mathrm{mmHg} E L N+/+$ and $E L N+/-$ aortae had similar extensibilities (23.6 \pm 0.9 vs. $22.5 \pm 0.7 \%$ ) (Fig. 2) (18). At $125 \mathrm{mmHg}$ and above, however, the pressure-diameter curves diverged with a marked reduction in extensibility of $E L N+/-$ aortae (at $125 \mathrm{mmHg}$, $18.8 \pm 1.5 \%$ for $E L N+/+$ aortae vs. $11.2 \pm 4 \%$ for $E L N+/-$ aortae, $P<0.05)$. The extensibility of $E L N+/-$ pulmonary arteries was similar to controls within a broad pressure range (0-50 $\mathrm{mmHg}$ ) surrounding normal physiologic pressure (mean pressure $=10 \mathrm{mmHg}$ ). These data indicate that $E L N+/-$ mice maintain extensibility at physiologic pressures.

To understand the structural determinants enabling ELN $+/-$ aortae to maintain normal extensibility at physiologic 
Table I. Number of Lamellar Units in ELN $+/+$ and

ELN + /- Aortae

\begin{tabular}{llccc}
\hline Mouse ID & Genotype & Age & Ascending aorta & Descending aorta \\
\hline BL6 & $E L N+/+$ & 5 mo & 9 & 6 \\
$390-3$ & $E L N+/+$ & 12 mo & 8 & 5 \\
$390-10$ & $E L N+/+$ & 14 mo & 8 & 6 \\
$390-11$ & $E L N+/+$ & 14 mo & 8 & 5 \\
$1002-7$ & $E L N+/+$ & 4 mo & 9 & 6 \\
$1029-8$ & $E L N+/+$ & 5 mo & 8 & 5 \\
$1045-3$ & $E L N+/+$ & 5 mo & 9 & 5 \\
& & Average & $8.4 \pm 0.5$ & $5.4 \pm 0.5$ \\
390 & $E L N+/-$ & 12 mo & 11 & 7 \\
$390-8$ & $E L N+/-$ & 14 mo & 11 & 7 \\
$390-9$ & $E L N+/-$ & 14 mo & 11 & 7 \\
$705-7$ & $E L N+/-$ & 7 mo & 11 & 8 \\
$720-2$ & $E L N+/-$ & 7 mo & 10 & 8 \\
$720-6$ & $E L N+/-$ & 7 mo & 10 & 6 \\
$1029-4$ & $E L N+/-$ & 6 mo & 11 & 8 \\
$1029-5$ & $E L N+/-$ & 6 mo & 10 & 7 \\
$1029-6$ & $E L N+/-$ & 5 mo & 10 & 7 \\
$1029-7$ & $E L N+/-$ & 5 mo & 10 & 7 \\
$1045-1$ & $E L N+/-$ & 6 mo & 11 & 7 \\
$1045-4$ & $E L N+/-$ & 6 mo & 10 & 8 \\
& & Average & $10.5 \pm 0.5$ & $7.3 \pm 0.6$ \\
& & & & \\
\hline
\end{tabular}

Differences in number of lamellar units between $E L N+/-$ mice and $E L N+/+$ mice were statistically significant, $P<0.005$.

pressure despite a $50 \%$ decrease in elastin, we examined the arterial structure. We discovered that aortae dissected from $E L N+/-$ mice (5-14 mo) had additional lamellar units. Consistent with previous work, we found that $E L N+/+$ aortae had 5.4 \pm 0.5 (descending) and 8.4 \pm 0.5 (ascending) layers of elastic lamellae (Table I and Fig. 3) $(10,19)$. By contrast, crosssections of $E L N+/-$ mice revealed an increase in the number of elastic lamellae to $7.3 \pm 0.6$ (descending) and $10.5 \pm 0.5$ (ascending) layers, respectively $(P<0.005)$. This represented an increase of $35 \%$ and $25 \%$ for the descending and ascending aortae of $E L N+/-$ mice, respectively. Similar changes were observed in the pulmonary artery with an average of $7.5 \pm 0.7$ lamellar units in $E L N+/-$ mice vs. $6.1 \pm 0.2$ in the $E L N+/+$ mice $(P<0.005)$. These changes were also apparent at birth. Our data demonstrate that $E L N+/-$ mice develop additional rings of elastic lamellae and smooth muscle. Thus, the number of lamellar units in an arterial wall is not fixed or species specific.

To determine if developmental changes observed in the aortae of $E L N+/-$ mice were also present in humans, we examined aortic segments from individuals with SVAS $(n=2$ for affected, $n=3$ for controls; Fig. 4 and data not shown). Previous studies focused only on arterial sections affected by discrete stenosis and showed subendothelial accumulation of cells, hypertrophy of smooth muscle, disruption of elastic fibers, and fibrosis $(12,13)$. We studied regions of the aorta that were free of discrete stenosis. The number of lamellar units in controls was consistent with previous reports $(10,20)$. By contrast, the aortic wall of individuals with SVAS was thicker and contained 2.5 -fold more lamellar units $(152 \pm 27.6$ vs. $62 \pm 8.7 ; P<$ $0.025)$. These data indicate that humans, like mice, respond to
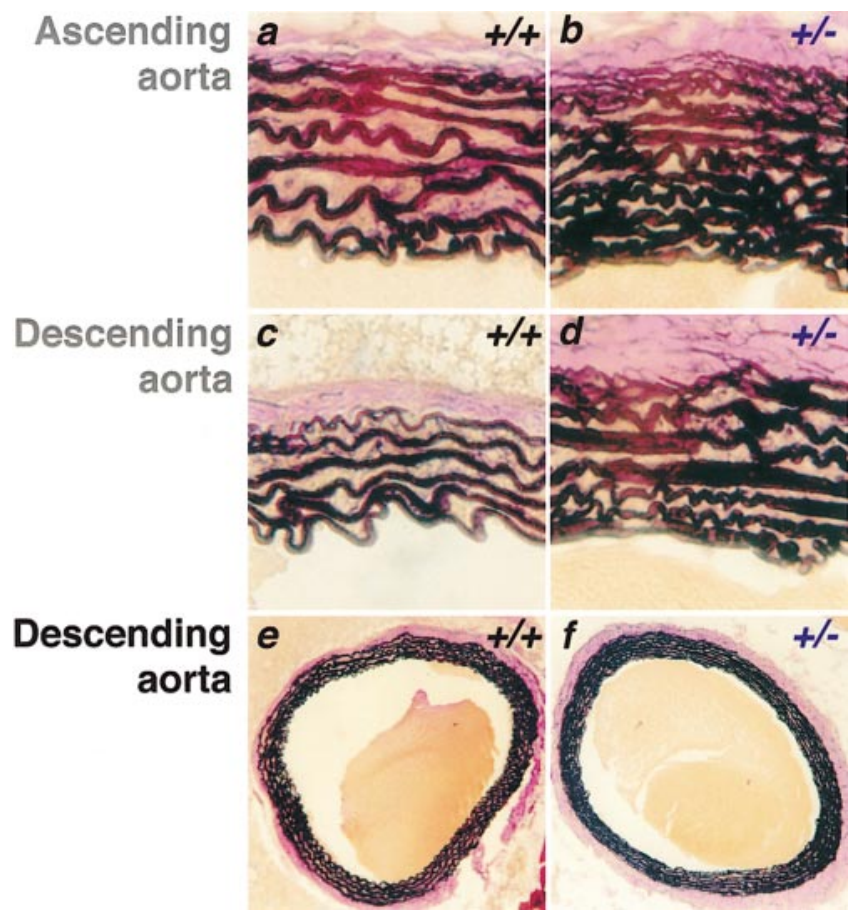

Figure 3. Hart stains of the ascending ( $a$ and $b)$ and descending $(c-f)$ aortae in $E L N+/+(a, c$, and $e)$ and $E L N+/-(b, d$, and $f)$ mice. Cross-sections are at the level where the pulmonary artery courses behind the ascending aorta. In this example there are 8 and 6 elastic lamellae in the ascending and descending aortae of the $E L N+/+$ mouse, respectively, compared with 11 and 8 in the $E L N+/-$ mouse, respectively, indicating an inverse relationship between $E L N$ expression and the number of elastic lamellae. Lower magnification of the descending aortae ( $e$ and $f$ ) demonstrates that the inner and outer diameters of $E L N+/+$ and $E L N+/$ - aortae are similar.

reduced elastin content during development by increasing the number of lamellar units.

\section{Discussion}

It was thought previously that the number of lamellar units in a developing arterial wall is fixed and species specific $(9,10)$. Our work demonstrates that this concept is incorrect. We found that $E L N+/-$ mice develop arteries with a $25-35 \%$ increase in the number of lamellar units. Examination of arterial specimens obtained from individuals with SVAS, a human disorder caused by ELN hemizygosity, revealed a 2.5 -fold increase in the number of elastic lamellae. Thus, the number of lamellar units in an arterial wall is not fixed or species specific and is modulated by $E L N$ expression.

Elastin's effect on arterial lamellar development likely involves smooth muscle cells sensing increased wall stress. The reduced $E L N$ mRNA and thinning of each elastic lamella observed in $E L N+/-$ mice would cause reduced extensibility in each lamella. These changes would lead to increased arterial wall stress, which is determined by arterial pressure and diameter and inversely proportional to the number of lamellar units and the tensile strength of each unit (21). However, the extensibility and diameter of $E L N+/-$ arteries were normal at physiologic pressures. Thus, mice with abnormal elastic fibers maintain arterial extensibility by increasing the number of 


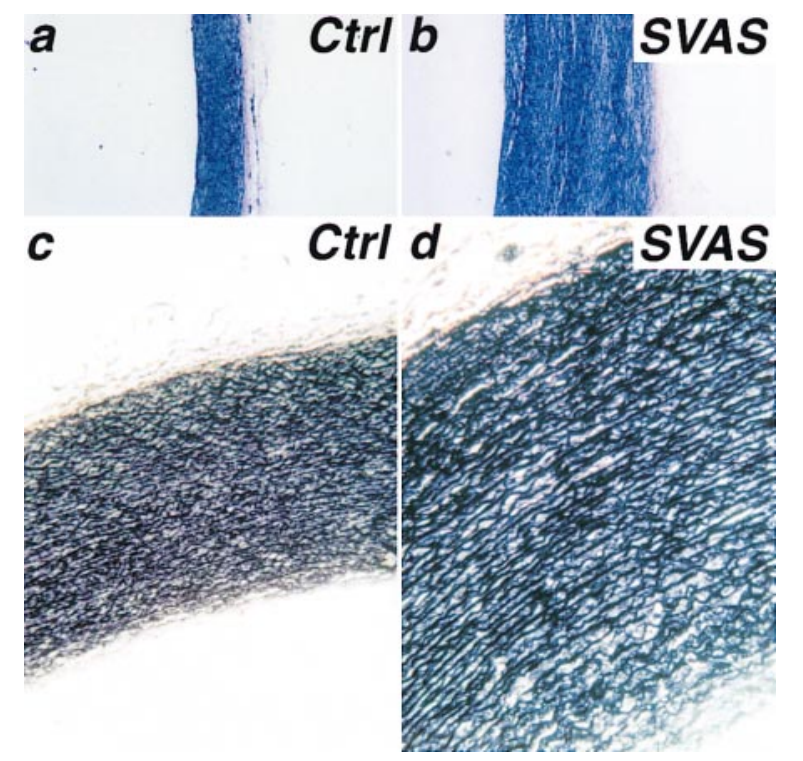

Figure 4. Elastin Van Gieson's stain of descending aortae cross-sections from a control $(a$ nd $c$ ) and a human with SVAS ( $b$ and $d$ ). Descending aortae were examined $1.0 \mathrm{~cm}$ distal to the left subclavian artery and were free of discrete stenosis. Low magnification of aortic sections $(a$ and $b)$ demonstrates the marked increase in thickness of SVAS samples. Higher magnification ( $c$ and $d$ ) shows 2.5 -fold more elastic lamellae in the aortae of an individual with SVAS, a disorder caused by ELN hemizygosity, indicating the sensitivity of humans to reduced developmental elastin.

lamellar units during development. Our model is further supported by anatomic and physiologic studies showing that the relationship between wall stress and the number of lamellar units in an artery is remarkably constant across species despite enormous variation in arterial diameter and stress (10). In addition, we observed no increase in the number of lamellar units when $E L N+/$ - aortae were cultured in the absence of hemodynamic stress (17 and data not shown). Thus, the physiologic force of wall stress is a key determinant of arterial development.

Our work defines a novel pathology in a human obstructive arterial disease, SVAS. Because of arterial pathology in ELN +/- mice, we examined aortic sections from individuals with SVAS and found a compensatory increase in arterial lamellar units. As human arteries are larger and wall stress is greater (110,000 dynes/cm in humans vs. 7,800 dynes/cm in mice) the increase in humans is profound (2.5-fold increase in humans). The increase in the smooth muscle cells needed to form additional layers likely outstrips blood supply, leading to medial necrosis and fibrosis. These changes in the medial wall would stimulate recurrent injury and repair resulting in the focal stenosis observed in SVAS $(12,13)$.

Our data indicate that the number of lamellar units can only be modulated by wall stress early in development. During gestation when pulmonary vascular pressures are similar to systemic pressures, pulmonary arteries respond to $E L N$ hemizygosity by increasing the number of lamellar units. Despite a sharp decrease in hemodynamic stress with onset of respiration at birth, the number of lamellar units in the pulmonary artery does not change. Later in life, lamellar structure is fixed and medial hypertrophy is the primary arterial response to increased hemodynamic stress (22). This concept is further sup- ported by the failure to observe increased lamellar units in other disorders associated with increased arterial wall stress or decreased elasticity like hypertension, aortic aneurysms, or Marfan's syndrome.

Other factors may also contribute to the risk of obstructive arterial disease by reducing $E L N$ expression during development. Hypervitaminosis D, for example, reduces $E L N$ expression in in vitro and in vivo systems $(23,24)$. Animal models exposed to hypervitaminosis D gave birth to offspring that developed SVAS $(25,26)$. These data support our model of reduced gestational ELN expression resulting in abnormal vascular development and obstructive vascular disease. Future studies need to define the impact of genetic and environmental modulators of $E L N$ expression on the risk of obstructive vascular disease.

\section{Acknowledgments}

We thank D. Atkinson for graphics; G. Moschini for help with manuscript preparation; K. Thomas, M. Capecchi, and M. Sanguinetti for critical comments; and K. Thomas and E. Eichwald for technical advice.

This work was supported by the National Institutes of Health, Bristol-Myers Squibb Foundation, and Fondation pour la Recherche Medicale.

\section{References}

1. Ross, R. 1993. The pathogenesis of atherosclerosis: a perspective for the 1990s. Nature. 362:801-809.

2. Ewart, A.K., C.A. Morris, D. Atkinson, W. Jin, K. Sternes, P. Spallone, A.D. Stock, M. Leppert, and M.T. Keating. 1993. Hemizygosity at the elastin locus in a developmental disorder, Williams syndrome. Nat. Genet. 5:11-16.

3. Curran, M., D. Atkinson, A. Ewart, C. Morris, M. Leppert, and M.T. Keating. 1993. The elastin gene is disrupted by a translocation associated with supravalvular aortic stenosis. Cell. 73:159-168.

4. Ewart, A., W. Jin, D. Atkinson, C. Morris, and M.T. Keating. 1994. Supravalvular aortic stenosis associated with a deletion disrupting the elastin gene. J. Clin. Invest. 93:1071-1077.

5. Olson, T.M., V.V. Michels, Z. Urban, K. Csiszar, A.M. Christiano, D.J. Driscoll, R.H. Feldt, C.D. Boyd, and S.N. Thibodeau. 1995. A 30kb deletion within the elastin gene results in familial supravalvular aortic stenosis. Hum. Mol. Genet. 4:1677-1679.

6. Li, D.Y., A.E. Toland, B.B. Boak, D.L. Atkinson, G.J. Ensing, C.A. Morris, and M.T. Keating. 1997. Elastin point mutations cause an obstructive vascular disease, supravalvular aortic stenosis. Hum. Mol. Genet. 6:1021-1028.

7. Lohler, J., R. Timpl, and R. Jaenisch. 1984. Embryonic lethal mutation in mouse collagen I gene causes rupture of blood vessels and is associated with erythropoietic and mesenchymal cell death. Cell. 38:597-607.

8. Dietz, H.C., and R.E. Pyeritz. 1995. Mutations in the human gene for fibrillin-1 (FBN1) in the Marfan syndrome and related disorders. Hum. Mol. Genet. 4:1799-1809.

9. Parks, W.C., R.A. Pierce, K.A. Lee, and R.P. Mecham. 1993. Elastin. Adv. Mol. Cell. Biol. 6:133-182.

10. Katoh, Y., and M. Periasamy. 1996. Growth and differentiation of smooth muscle cells during vascular development. Trends Cardiovasc. Med. 6 : $100-106$.

11. Wolinsky, H., and S. Glagov. 1967. A lamellar unit of aortic medial structure and function in mammals. Circ. Res. 20:99-111.

12. O'Connor, W. 1985. Supravalvular aortic stenosis: clinical and pathologic observations in six patients. Arch. Pathol. Lab. Med. 109:179-185.

13. Perou, M. 1961. Congenital supravalvular aortic stenosis. Arch. Pathol. Lab. Med. 71:113-126.

14. Davis, E.C. 1995. Elastic lamina growth in the developing mouse aorta. J. Histochem. Cytochem. 43:1115-1123.

15. Boyle, W.A., and G.M. Maher. 1995. Endothelium-independent vasoconstricting and vasodilating actions of halothane on rat mesenteric resistance blood vessels. Anesthesiology. 82:221-235.

16. Milnor, W.R. 1990. Cardiovascular Physiology. Oxford University Press, New York. 550 pp.

17. Li, D.Y., B. Brooke, E.C. Davis, R.P. Mecham, L.K. Sorensen, B.B. Boak, E. Eichwald, and M.T. Keating. 1998. Elastin is an essential determinant of arterial morphogenesis. Nature. 393:276-280.

18. Svendsen, U.G., C.M. Koch, and B. Rubin. 1979. Genetic control of the 
spontaneous hypertension in the NZB/Cr strain of mice. Immunogenetic considerations. APMIS. 87:269-273.

19. Davis, E.C. 1993. Smooth muscle cell to elastic lamina connections in developing mouse aorta. Role in aortic medial organization. Lab. Invest. 68:89-99.

20. Machii, M., and A.E. Becker. 1997. Hypoplastic aortic arch morphology pertinent to growth after surgical correction of aortic coarctation. Ann. Thorac. Surg. 64:516-520.

21. Wolinsky, H., and S. Glagov. 1964. Structural basis for the static mechanical properties of the aortic media. Circulation. 14:400-413.

22. Churg, J., and M.H. Goldstein. 1995. Pathologic aspects of hypertension. In Vascular Pathology. W.E. Stehbens and J.T. Lie, editors. Chapman and
Hall, London. 571-580.

23. Vijayakumar, S.T., and P.A. Kurup. 1974. Hypervitaminosis D and glycosaminoglycan metabolism in rats fed normal and high fat cholesterol diets. $J$. Nutr. 104:423-429.

24. Hinek, A., M.D. Botney, and R.P. Mecham. 1991. Inhibition of tropoelastin expression by 1,2 dihydroxyvitamin D3. Connect. Tissue Res. 26:155-166.

25. Friedman, W.F., and W.C. Roberts. 1966. Vitamin D and supravalvular aortic stenosis syndrome. Circulation. 34:77-86.

26. Chan, G.M., J.J. Buchino, D. Mehlhorn, K.E. Bove, J.J. Steichen, and R.C. Tsang. 1979. Effect of vitamin D on pregnant rabbits and their offspring. Pediatr. Res. 13:121-126. 